\title{
Real-Time Traffic Light Detection and Interpretation Using Circle Centroid
}

\author{
Zamani Md Sani, Nur Amalyna Ramlan
}

\begin{abstract}
The paper proposes a new method to recognize the sequence of a traffic light using image processing algorithm. Invariant in factor lightning and weather condition that lead to misinterpret the color of traffic light is one of the factors of accident at traffic light conjunction besides the behavior of the driver itself. The process to identify the color and shape of traffic light are Image Acquisition, Pre-Processing, Detection, Feature Extraction and Interpretation. RGB normalization is performed and simple thresholding method that acts as color segmentation provides a better division of the traffic light colors. Circle Hough Transform and HSV color features based on the traffic light aspect are used to decide whether the spots on the frames are likely to be traffic lights' color and shape. The detection of traffic light will be obtained after identifying the feature such as the centroid of the Circle Hough Transform that need to be extracted at the end of the result. The research has been improved by focusing on detection and interpretation of traffic light based on real time video rather than image sample as the input. The proposed algorithm can detect the green color accurately with maximum accuracy of $83.8 \%$, yellow color of $75.6 \%$ and red color with minimum accuracy of $70.19 \%$. This indicates that there is a possibility to use the proposed algorithm to detect the three different color of green, yellow and red color of traffic lights' colour.
\end{abstract}

Keywords: Circle Hough Transform Centroid, RGB normalization, traffic light.

\section{INTRODUCTION}

Traffic light is a signaling device to make sure the flow of traffic is controlled smoothly. There are three state of traffic lights that are red, yellow and green. The standard structure of traffic light's is bounding box comprises of red light above followed by yellow and green. Some of the traffic signal is mounted horizontally or sideways. All the drivers need to be alerted with the flashing color of green, amber and red of traffic light as shown as in Fig. 1. Traffic light is designed normally at the busiest street and highway intersection to achieve a purpose of facilitating the safe movement of cars and avoiding car collisions. Notwithstanding, The Federal Highway Administrations guarantees that around $45 \%$ of auto accidents occur at a street crossing point and the reason is normally identified with running a stop sign. It appears that a large portion of them are caused by human mistake [1].

Revised Manuscript Received on September 14, 2019.

Zamani Md Sani, Mechatronics Department, Durian Tunggal, Melaka, Malaysia. (Email: zamanisani@utem.edu.my)

Nur Amalyna Ramlan, Mechatronics Department, Durian Tunggal, Melaka, Malaysia.

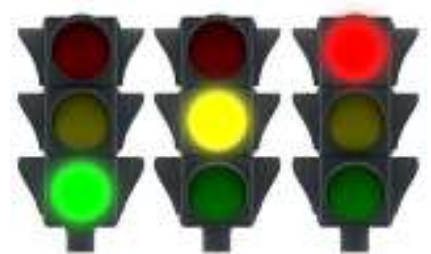

Fig. 1.State of traffic light.

Autonomous car industry are getting more attention is this decade where the detection of traffic light color is the focal point in autonomous car [2]-[4]. This type of detection system is more reliable and faster which will be a great solution of producing zero accidents as it will control the car automatically and efficiently to obey the traffic light sign. Apart of protecting drivers from death and injury, it also eliminates property damage, reduces traffic problems and could bring down car insurance rates [5]. It may also help people with color blind disabilities and the elderly [6]. Previous research used color space conversion to Hue, Saturation Value (HSV) [7], binarization and morphology features filtering methods in the preprocessing stage. However, there are some improvements in later where they use image segmentation, morphology process and threshold segmentation in $\mathrm{YCbCr}$ color space [8], [9]. The feature extracted for example the Gabor wavelet transform and 2D independent component analysis (2DICA) use for the recognition process. Methods such as down-sampling, circular regions detection and modified Hough transform were used for the next process. After the feature extraction had been completed, it will involve the process of recognition, such as using the K-nearest neighbor and pixel matching [10]. Fuzzy clustering also provides a better division of the traffic light [11].

In this research, the detection is based on the flashing traffic light color rather than other dim color. So it is significant to determine the pixel intensity of each traffic color correctly as to differentiate between bright or dim color of the traffic light. The flashing or bright color of traffic is not determined by the brightness, but it is totally depended by the amount of intensity of the color. Intensity refers to the measure of light or the numerical estimation of a pixel while brightness relies upon visual perception when comparing with a reference. RGB normalization is applied to get the best segmentation process for the traffic light color. Also the recognition of the light is based on the pixel value on the centroid location of the circle detected. 


\section{METHODOLOGY}

\section{A Hardware Implementation}

A USB camera was used to face straight ahead and is mounted to rear view mirror. At this point, the camera will have wider field of detection and can detect the color of the traffic light earlier at certain fixed distance without any disturbance. The location of the camera setup is shown as in Fig. 2.

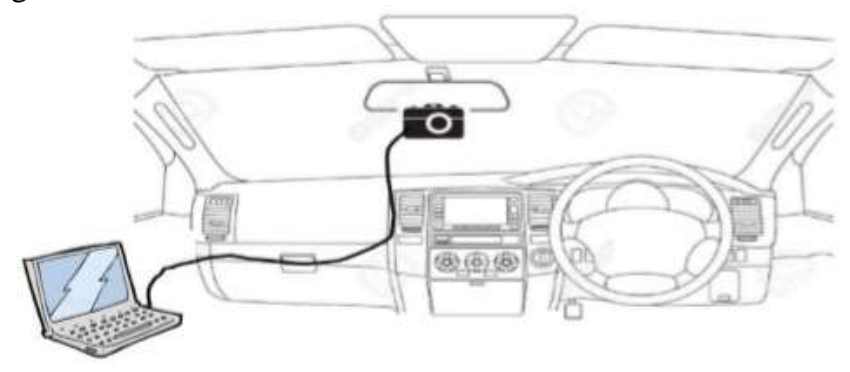

Fig. 2.Camera setup.

Author (s) can send paper in the given email address of the journal. There are two email addresses. It is compulsory to send paper in both email address.

\section{B Image Processing}

The preprocessing process for the image will be applied to get a better image. RGB samples were selected from constant atmospheric conditions that are sunny day (early mornings and early evening) during the day time with a good illumination only. RGB color space is sensitive to the light intensity. So, in order to uniform the drift in colors, the color space will be converted later to get a better filtering process.

RGB normalization process is then used because RGB normalized color space is quite invariant to lighting change and object geometry, being robust to lighting conditions. It supports more distinguishable colors for traffic light lamps. To perform RGB normalization the individual color channels are summed and each channel is divided by the sum such as in equations below.

$$
\begin{aligned}
& (x, y)=(\mathrm{R}, \mathrm{G}, \mathrm{B}) \\
& S=R+G+B \\
& R=\frac{R}{S} \times 255 \\
& G=\frac{G}{S} \times 255 \\
& B=\frac{B}{S} X 255
\end{aligned}
$$

If $\mathrm{S}=0$, then $\mathrm{R}=\mathrm{G}=\mathrm{B}$ where $\mathrm{S}=$ Total, $\mathrm{R}, \mathrm{G}, \mathrm{B}=$ pixel values and $255=$ scaling factor.

The process will blur the image and bright certain important parts only. When normalizing the RGB values of an image, each pixel's value in an image is divided by the sum of the pixel's value over all channels. Normalized RGB color threshold removes most of the elements in the scene that are not possible candidates for being a traffic light.

One of the important steps in this system is color segmentation which act to discriminate color states among traffic light. It streamlines the vision problem by assuming that objects are hued distinctively, and that gross shading contrasts that matter. As a result, the image can be processed very rapidly after getting rid the information about color and brightness variations that provides many valuable cues about the shapes and textures of 3D surfaces from the image. In this research, simplest color segmentation method used is called the thresholding method. This method is based on a clip-level (or a threshold value) to turn a grayscale image into a binary image. The specific threshold value will be determined to detect the three different possible color of traffic light. Saturation spots, relating to lenses center or false alarms in building, trees and sunshine will be the main challenges in this color segmentation process. Each sample image taken has its own different type and amount of pixels.

Morphological operations referring to was performed based on erosion and a dilation to act on the red, amber and green traffic light images will be composed in this stage and then filtering rules will take after the process. It is carried out to get rid little conceivable elements in the background by removing some pixel that is not important in erosion process as in (6). Dilation process will add some pixels to the pixels left in the image after going through the erosion process as in (7). This process will re-establish the original size of the blobs and make them more compact.

Dilation

$$
\begin{aligned}
& A \oplus B=\{z \in E \mid(B 8) z \cap A \neq \emptyset\} \\
& \text { Erosion }
\end{aligned}
$$$$
A \oplus B=\{z \in E \mid B z \subseteq A\}
$$

In summary, the preprocessing processes involved as in Fig. 3.

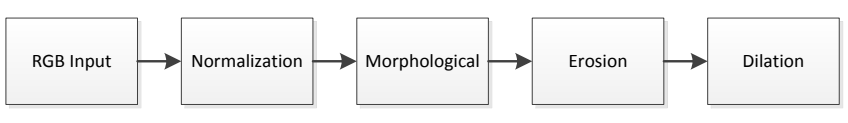

Fig. 3.Preprocessing process.

\section{Feature Extraction and Recognition Process}

To get better features for the extraction, The RGB image is converted to HSV color space - hue, saturation. Other than the hue, the pixel intensity for the specific color of traffic light is determined. Only dominant hue for all those colors is extracted together with its average brightness and saturation values. This process will determine the accurate color of traffic light and prevent detecting the false positive colors like street light lamps and car brake's lamps.

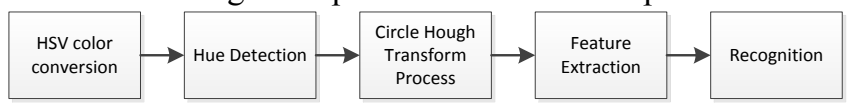

\section{Fig. 4.Process prior to feature extraction and recognition.}

Circle Hough Transform [12] is applied to the image to detect circular shape of the traffic light and its center coordinates. A complete circle of radius, $r$ is created when the $\theta$ change from 0 to 360 degrees. The radius of circles shape of traffic light color is fixed. All the above equations will be applied to the loaded image in MATLAB software. Edges will be detected and producing a binary image. Circle is produced in the $(a, b)$ space from every of edge of the pixel. After that, cast will 'vote' those point of circle in the (a, b) space in the accumulator cells and the cells with greater number of votes will concluded as the center of 
circle as shown in Fig. 5. The determined center coordinate will make it easier to clarify the pixel value of the traffic light's color. The pixel value of each color of traffic light is depended primarily on the coordinate of the center. The pixel value is being compared by several radius of traffic light's circle shape.

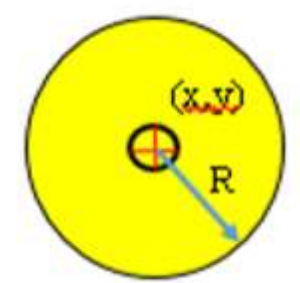

Fig. 5.Circle of the traffic light.

In this new approach the pixel value of each color of traffic light is depended primarily on the coordinate of the center which is why by determining the center coordinate will make it easier to clarify the pixel value of the traffic light's color for the recognition as in Fig. 5. Later, the value for maximum, intermediate and minimum radius are being recorded and observed.

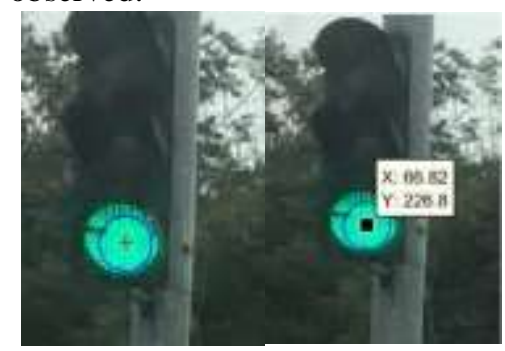

Fig. 6.Applying the Circle Hough Transform and its center coordinate.

Finally, image matching process is used to identify the state of the traffic light. Area of pixels that contained in the traffic light's blob is determined. There will be a reference image which will be compared pixel by pixel to the real time image project for decision making. Images will be matched if it has same pixel values. The entire region that contain almost the same amount of pixel value and circle shape will later represent the entire traffic light, which accomplishes the recognition of traffic light color and its circle shape.

\section{RESULTS AND DISCUSSION}

The image results from the full process (from detecting the traffic light until determining the status of the color of the traffic light) can be shown as in Fig. 7(a)-(c). Image from 7.1(a), 7.2(a) and 7.3(a) is from the original video images. It will go through the RGB normalization process as in 7.1 (b), 7.2(b) and 7.3 (b). This process is done to remove the change in illumination of the original image. It will reduce the effects of light, removes highlighted regions and shadows which make that object easier to be detected. After that, the normalized image will be processed by erosion and dilation method in Fig. 7.1(c), 7.2(c) and 7.3(c). This process will remove noise at the image background to increase accuracy for classification process. Three new filtered images were created relating to the three traffic light colors. After that, the image will be extracted to find the feature of traffic light which is color and circle detection.
The color feature will be extracted using the HSV color space which is shown in Fig. 7.1(d), 7.2(d) and 7.3(d) to classify accurately whether the detected color is green, yellow or red based on its pixel value. There are possibilities for false positive colors like street light lamps and car brake's lamps to be occurred can be reduced in this process.

To improve the detection process, the circle shape of traffic light is detected in Fig. 7.1(e), 7.2(e) and 7.3(e) and the centroid of the circle is identified. Pixel matching process will clarify whether green, yellow and red traffic light's color is detected in the real time video.
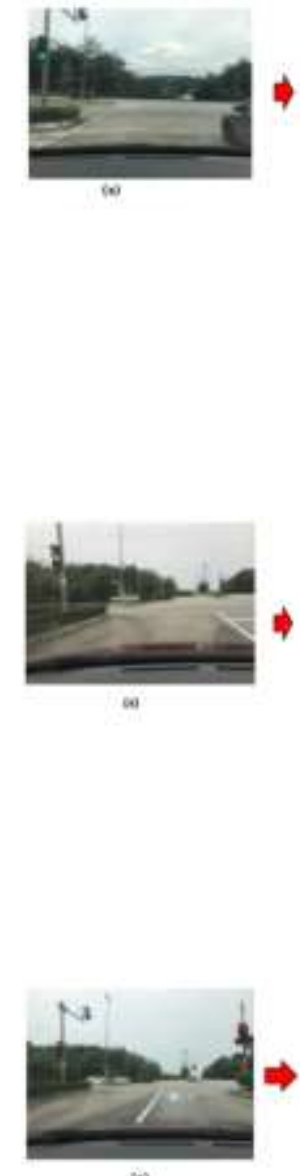

(i)
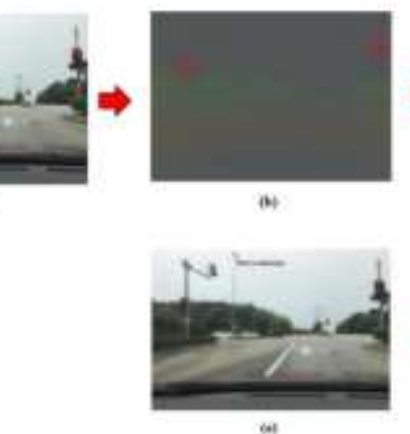

7.3

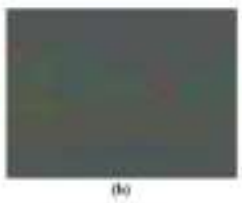

(a)

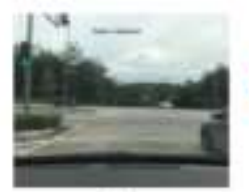

(6)

7.1

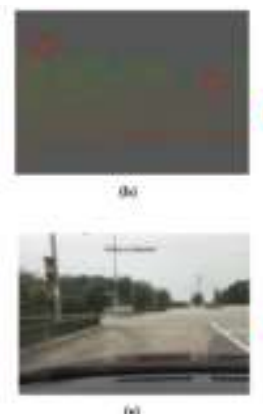

7.2

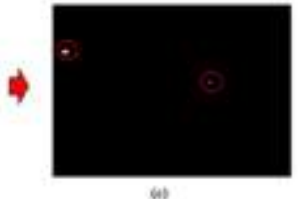

Q

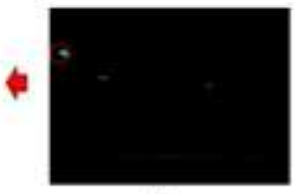

(6)

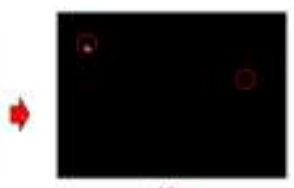

$+6$

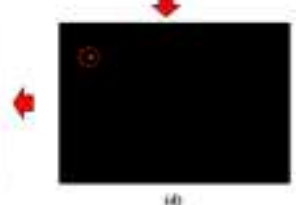

(i)

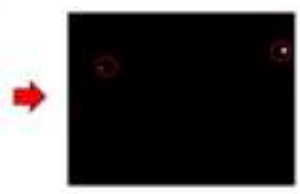

ic

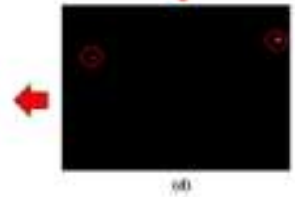

(4)
Fig. 7.Complete Cycles for the detection and recognition of the traffic light color (a) Green (b) Amber and (c) Red.

The accuracy of each color of traffic light is determined by examining the total number of frame of video that has been processed as in (8) and (9).

Percentage of accuracy $=1$ - classifation error

Classification error, $E=f / n \times 100 \%$

where

$\mathrm{f}=$ number of incorrectly classified samples (false positive + false negative)

$\mathrm{n}=$ number of samples

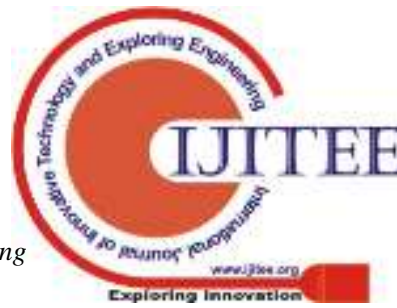


The traffic light's color is detected in each frame which will produce false positive and false negative as the result. The false negative is the incorrectly detected of traffic light color which may occur due to some factors. For example, Table I shows the accuracy for all traffic light color.

Table- I: Accuracy table for different part of traffic light color (a) Green (b) Amber (c) Red

\begin{tabular}{|l|l|l|l|l|}
\hline Video & $\begin{array}{l}\text { No of } \\
\text { Frames }\end{array}$ & $\begin{array}{l}\text { True } \\
\text { Positive }\end{array}$ & $\begin{array}{l}\text { False } \\
\text { Positive }\end{array}$ & Accuracy \\
\hline Vid_1 & 252 & 207 & 45 & $82.1 \%$ \\
\hline Vid_2 & 264 & 225 & 39 & $85.2 \%$ \\
\hline Vid_3 & 208 & 148 & 28 & $84.0 \%$ \\
\hline Average & & & & $83.8 \%$ \\
\hline
\end{tabular}

(a)

\begin{tabular}{|l|c|c|c|c|}
\hline Video & $\begin{array}{c}\text { No of } \\
\text { Frames }\end{array}$ & $\begin{array}{c}\text { True } \\
\text { Positive }\end{array}$ & $\begin{array}{c}\text { False } \\
\text { Positive }\end{array}$ & $\begin{array}{c}\text { Accur } \\
\text { acy }\end{array}$ \\
\hline Vid_1 & 174 & 137 & 37 & $78.7 \%$ \\
\hline Vid_2 & 105 & 74 & 31 & $70.4 \%$ \\
\hline Vid_3 & 149 & 116 & 28 & $77.8 \%$ \\
\hline Average & \multicolumn{4}{|l}{} \\
\hline
\end{tabular}
(b)

\begin{tabular}{|l|c|c|c|c|}
\hline Video & $\begin{array}{c}\text { No of } \\
\text { Frames }\end{array}$ & $\begin{array}{c}\text { True } \\
\text { Positive }\end{array}$ & $\begin{array}{c}\text { False } \\
\text { Positive }\end{array}$ & $\begin{array}{c}\text { Accur } \\
\text { acy }\end{array}$ \\
\hline Vid_1 & 293 & 196 & 97 & $66.9 \%$ \\
\hline Vid_2 & 198 & 145 & 53 & $73.2 \%$ \\
\hline Vid_3 & 237 & 167 & 70 & $70.5 \%$ \\
\hline Average & & & \multicolumn{2}{|c|}{} \\
& & & 70.19 \\
\hline
\end{tabular}

(c)

One of the main factors resulting in false positive is due to the illumination and light intensity which on cloudy and foggy day the accuracy will be a bit lower in detection. In addition, the reflecting lights from the surrounding such as the metal from the cars and the interval between the light changes may also causing the accuracy to be lower. The flashing color of the traffic light will become too saturated and turn dimmer which affecting their actual RGB value. This need to be avoided because it may produce some sort of distortion which may interfere the measurement of bright regions of scene because every recorded image is limited to some maximum value.

From the results of green traffic light detection and interpretation using image processing method has achieved the highest accuracy, while red traffic light has achieved the lowest percentage in term of accuracy. It can be said that the detection on green traffic light can be made effectively even at a far distance compared to the other colors. The large amount of false positive especially on the red light colour is due to several factors such as the distance of the camera and the traffic light which may contribute to lighter color which resembles orange and, the presence of the other car's circle brake lamps which may have the same color and may affect the detection process too.

\section{CONCLUSION}

The paper presented a new approach for traffic light detection and interpretation. The approach is additional to find the centroid coordinate of the circle, detected after the process of Circle Hough Transform. With the coordinate, further verification of the traffic light status is performed. Based on the accuracy result, red color performs the best accuracy while green perform the lowest accuracy and possible factors had been discussed as above such as interference of the same color and shape from the surrounding. Future works should include improving the detection of the traffic light and the recognition of the lighter color due to the longer range between the camera and the traffic light.

\section{ACKNOWLEDGEMENT}

The authors would like to acknowledge the funding support received from Pusat Pengurusan Penyelidikan dan Inovasi, CRIM Universiti Teknikal Malaysia Melaka (UTeM).

\section{REFERENCES}

1. U.S. Department of Transportation. (2018). Intersection safety-Background and objectives. [Online]. Available: https://www.fhwa.dot.gov/research/topics/safety/intersect ions/.

2. S. K. Sivarao, M. Esro, and T. J. Anand, "Electrical and mechanical fault alert traffic light system using wireless technology," International Journal of Mechanical and Mechatronics Engineering, 10(4), 2010, pp. 19-22.

3. A. Saà, Traffic lights recognition in images for intelligent vehicles. [Online]. Available: https://www.researchgate.net/profile/Albert_SaaGarriga/publication/272088763_Master_Thesis_Traffic_ Lights_Detection_in_Images_for_Intelligent_Vehicles/li nks/54da2e0c0cf25013d04471fd.pdf.

4. V. Goel, S. Singhal, T. Jain, and S. Kole, "Specific color detection in images using RGB modelling in MATLAB," Int. J. Comput. Appl., 161(8), 2017, pp. 38-42.

5. V. R. Gannapathy, S. K. Subramaniam, A. B. Mohamad Diah, M. K. Suaidi, and A. H. Hamidon, "Risk factors in a road construction site," Proceedings of the World Academy of Science, Engineering and Technology, 46, 2008, pp. 640-643.

6. A. Abdelfatah. (2016). Traffic fatality causes and trends in Malaysia. [Online]. Available: https://malaysiacities.mit.edu/sites/default/files/document s/Abdelfatah.pdf.

7. Z. Ozcelik, C. Tastimur, M. Karakose, and E. Akin, "A vision based traffic light detection and recognition approach for intelligent vehicles," IEEE International Conference on Computer Science and Engineering, 2017, pp. 424-429.

8. Z. Cai, M. Gu, and Y. Li, "Real-time arrow traffic light recognition system for intelligent vehicle," International Conference on Image Processing, Computer Vision, and Pattern Recognition, 2012, pp. 1-7.

9. Z. Cai, Y. Li, and M. Gu, "Real-time recognition system of traffic light in urban environment," IEEE Symposium on Computational Intelligence for Security and Defence Applications, 2012, pp. 1-6.

10. J. L. Binangkit and D. H. Widyantoro, "Increasing accuracy of traffic light color detection and recognition using machine learning," IEEE 10th International Conference on Telecommunication Systems Services and Applications, 2016, pp. 1-5.

11. M. Diaz-Cabrera, P. Cerri, and P. Medici, "Robust realtime traffic light detection and distance estimation using a single camera," Expert Syst. Appl., 42(8), 2015, pp. 3911-3923.

12. C. Tomasi, "Histograms of oriented gradients," Comput Vis. Sampl., 2012, pp. 1-6. 


\section{AUTHORS PROFILE}

Zamani Md Sani received his Bachelor and Master in Electrical and Electronics Engineering from University Sains Malaysia in 2000 and 2009 Currently, he is pursuing his Ph.D. degree from MMU and also a staff member at Universiti Teknikal Malaysia Melaka.

NorAmalyna completed her degree in 2019 and currently working in the industry as an engineer. 